\title{
Construction of Sc Chordal and Sc Weakly Chordal Graphs
}

\author{
Parvez Ali ${ }^{1}$, Syed Ajaz Kareem Kirmani ${ }^{2}$ \\ College of Engineering, Unayzah, Qassim University, Al-Qassim, Kingdom of Saudi Arabia
}

Email address:

parvezamu@rediffmail.com (P. Ali), ajazkirmani@rediffmail.com (S. A. K. Kirmani)

\section{To cite this article:}

Parvez Ali, Syed Ajaz Kareem Kirmani. Construction of Sc Chordal and Sc Weakly Chordal Graphs. American Journal of Applied Mathematics. Vol. 4, No. 3, 2016, pp. 163-168. doi: 10.11648/j.ajam.20160403.17

Received: April 13, 2016; Accepted: May 18, 2016; Published: June 4, 2016

\begin{abstract}
Study of any graph class includes characterization, recognition, counting the number of graphs i.e. cataloging and construction of graphs. The construction of sc chordal graphs by mean of complementing permutation is one of the known method. In this paper, a new method for the construction of sc chordal graphs is proposed based on a two-pair of graphs. We also presented algorithm for the construction of sc weakly chordal graphs.
\end{abstract}

Keywords: Self-Complementary Graph, Chordal and Weakly Chordal Graph, Two-Pair, Degree Sequence, $\mathrm{P}_{4}$

\section{Introduction}

Let $G=(V, E)$ be an undirected graph with no loops or multiple edges where $V(G)$ and $E(G)$ denote the set of vertices and edges of $G$ respectively. Let $S \subseteq V(G)$ be a set of vertices of $G$; the subgraph of $G$ induced by $S$ is denoted by $G[S]$. The neighborhood $N(x)$ of a vertex $x \in V(G)$ is the set of all the vertices of $G$ which are adjacent to $x$. A path $v_{0}$, $v_{1}, \ldots, v_{\mathrm{k}}$ of a graph is called simple if none of its vertices occurs more than once; it is called a cycle(simple cycle) if $v_{0} v_{\mathrm{k}} \in E(G)$. A simple path (cycle) is chordless if $v_{i} v_{j} \notin E(G)$ for any two non-consecutive vertices $v_{\mathrm{i}}, v_{\mathrm{j}}$ in the path (cycle). A chordless path (chordless cycle) on $n$ vertices is denoted by $P_{\mathrm{n}}\left(C_{\mathrm{n}}\right)$. Let $G$ be a graph with an induced $P_{4}[a, b, c, d]$, vertices $b, c$ are called midpoints of the $P_{4}$ and $a, d$ are called endpoints of the $P_{4}$. The degree sequence of a graph $G$ is the sequence of the degrees of the $n$ vertices of $G$ arranged in non-increasing order and is denoted by $d_{1} \geq d_{2} \geq \ldots \geq d_{\mathrm{n}}$. A pair $\{x, y\}$ of non-adjacent vertices such that every chordless path from $x$ to $y$ has exactly two edges is known as two-pair. A co pair is a complement of a two pair. Let $n$ be the number of vertices and $m$ be the number of edges in graph $G$.

A graph is self-complementary (sc) if it is isomorphic to its complement. Every sc graph has $4 p$ or $4 p+1$ vertex, where $p$ is a positive integer. A graph $G$ is chordal if it has no chordless cycle of length greater than or equal to 4 and it is weakly chordal if both $G$ and its complement $\bar{G}$ have no chordless cycle of length greater than or equal to 5 . A self-complementary graph which is also chordal (respectively, weakly chordal) is called sc chordal graph (resspectively sc weakly chordal graph). For other definitions we refer to [6].

The construction problem of sc graphs is a fundamental problem in studying sc graphs. The construction of sc graphs by mean of complementing permutation was considered in [4] and [5]. In [7] Jin and Wong proposed the decomposition method for the construction of sc graphs. Gibbs [11] has given algorithms for the construction of sc graphs with $4 p$ and $4 p+1$ vertices. Sridharan and Balaji [10] also gave algorithms for the construction of sc chordal graphs with $4 p$ and $4 p+1$ vertices by modifying the algorithm as discussed in [11].

There are several techniques discussed in [2], [8] and [13] for the construction of a graphs having some specific properties. In [13] X. Huang and Q. Huang gave a method to construct many classes of connected graphs with exactly kmain eigenvalues for any positive integer $\mathrm{k}$. In [8] Lihuan Mao et. al gave a new method for the construction of graphs by using their generalized spectrum. Recently Honag et. al in [2] discussed construction of k-critically $\mathrm{P}_{5^{-}}$free graphs.

In this note we study the construction problem for the subclasses of sc perfect graphs, namely sc chordal graphs and sc weakly chordal graphs. Section 2 is devoted to sc chordal graphs where a construction algorithm for sc chordal graphs is propose. In section 3 we study sc weakly chordal graphs and present an algorithm for the construction of sc weakly chordal graphs. 


\section{Construction of Sc Chordal Graphs}

In this section we propose a different approach for the construction of sc chordal graph using the concept of twopair. Fulkerson and Gross [3] gave the following classical construction scheme for chordal graphs, which works as follows: "Start with a graph $G_{0}$ with no vertices and repeatedly add a vertex $v_{\mathrm{j}}$ to $G_{\mathrm{j}-1}$ to create the graph $G_{\mathrm{j}}$ such that $v_{\mathrm{j}}$ is not the middle vertex of any $P_{3}$ of $G_{\mathrm{j}}$ '”.

This construction scheme for chordal graphs is known as a vertex addition scheme. Now for obtaining the different method for the construction of sc chordal graphs we need the following results.

Theorem 1 [9]. Let $G$ be a sc graph with degree sequence $d_{1} \geq d_{2} \geq \ldots \geq d_{n}$. Then $G$ is chordal graph iff $\sum_{i=1}^{2 p} d_{i}=6 p^{2}-2 p$ for $n=4 p \& \sum_{i=1}^{2 p} d_{i}=6 p^{2}$ for $n=4 p+1$.

Theorem 2. [1]. Let $G_{1}$ be a chordal graph and $\{x, y\}$ be a pair of non-adjacent vertices of $G_{1}$. Let $G_{2}$ be the graph obtained from $G_{1}$ by adding edge $x y$; then $G_{2}$ is chordal iff $\{x, y\}$ is a two-pair of $G_{1}$.

Using Theorem-2, We propose the following algorithm-1 for the construction of sc chordal graph with the given degree sequence $\left(d_{1} \geq d_{2} \geq \ldots \geq d_{n}\right)$. We start with a graph $\left(G_{\mathrm{i}}\right)$ having all $n$ isolated vertices and repeatedly add an edge between the non-adjacent vertices ( $x$ and $y$ ) to create the graph $\left(G_{\mathrm{i}+1}\right)$ such that $\{x, y\}$ is a two-pair in $G_{\mathrm{i}}$. Algorithm-1 repeats this process until required degree sequence is obtained.

The following algorithm constructs sc chordal graph with the given degree sequence on $n$ vertices.

Algorithm 1. An Algorithm for the construction of sc chordal graph.

Input: A degree sequence $\left(d_{1} \geq d_{2} \geq \ldots \geq d_{n}\right)$ of sc graph.

Output: A sc chordal graph with degree sequence $\left(d_{1} \geq d_{2} \geq \ldots \geq d_{n}\right)$.

Step-1: Start with a graph $G_{1}$ with $n$ vertices and no edges.

Step-2: Add an edge between two non-adjacent vertices ( $x$ and $y$ ) of $G_{1}$, to. obtain graph $G_{2}$ such that $\{x, y\}$ is a two-pair in $G_{1}$.

Step-3: Repeat the process of step-2, until we get a graph with degree sequence $\left(d_{1} \geq d_{2} \geq \ldots \geq d_{n}\right)$.

End.

The following Theorem states that a graph $G$ with $n$ vertices is a sc chordal iff it can be constructed by algorithm-1

Theorem 3.

(i). The constructed graph by algorithm-1 is a sc chordal graph with $\left(d_{1} \geq d_{2} \geq \ldots \geq d_{n}\right)$ degree sequence.

(ii). Every sc chordal graph with $\left(d_{1} \geq d_{2} \geq \ldots \geq d_{n}\right)$ degree sequence on $n$ vertices can be constructed by algorithm- 1 .

Proof.

(i). The algorithm-1 takes degree sequence $\left(d_{1} \geq d_{2} \geq \ldots \geq d_{n}\right)$ of sc graphs as input and it construct chordal graphs from empty graphs using Theorem-2, which ensures chordality. Also from Theorem-1, no other graph is possible with the same degree sequence.

(ii). Follows from Theorem- 2.

We illustrate algorithm-1 by constructing a sc chordal graph on 12 vertices.

Suppose we have to obtain a sc chordal graph on 12 vertices with degree sequence $(10,10,8,8,6,6,5,5,3,3,1,1)$. Algorithm-1 starts with an empty graph $G_{1}$ on 12 vertices $\left\{v_{1}\right.$, $\left.v_{2}, v_{3}, v_{4}, v_{5}, v_{6}, v_{7}, v_{8}, v_{9}, v_{10}, v_{11}, v_{12}\right\}$ as shown in figure-1(a). The overall procedure of the construction of sc chordal graph with the given degree sequence $(10,10,8,8,6,6,5,5,3,3,1$, 1) is obtained as given below and illustrated in figure-1

(i). The graph $G_{2}$ as shown in figure-1(b) can be easily obtained, since in $G_{1}$ there is no edge and in the given degree sequence there is a vertex of degree 10 . So we select any vertex (let it be $v_{1}$ ) arbitrarily to give it adjacency 10 by adding edges $e_{1}, e_{2}, e_{3}, e_{4}, e_{5}, e_{6}, e_{7}$, $e_{8}, e_{9}, e_{10}$ from vertex $v_{1}$ to $v_{2}, v_{3}, v_{4}, v_{5}, v_{6}, v_{7}, v_{8}, v_{9}$, $v_{10}$ and $v_{11}$ respectively. In this way we get graph $G_{2}$ as shown in figure-1(b). Obviously there is no violation of step- 2 of algorithm-1 by adding these edges in this way.

(ii). In the given degree sequence we have another vertex of degree 10. Let this vertex be $v_{2}$. Since $v_{2}$ already got 1 adjacency as can be seen in figure-1(b). So we give it 9 more adjacencies by adding edges $e_{11}, e_{12}$, $e_{13}, e_{14}, e_{15}, e_{16}, e_{17}, e_{18}$ and $e_{19}$ from vertex $v_{2}$ to $v_{3}, v_{4}$, $v_{5}, v_{6}, v_{7}, v_{8}, v_{9}, v_{10}, v_{12}$ respectively. In this way we obtain graph $G_{3}$ as shown in figure-1(c). From figure1 (b) it is clear that all the non-adjacent pairs i.e. $\left\{v_{2}\right.$, $\left.v_{3}\right\},\left\{v_{2}, v_{4}\right\},\left\{v_{2}, v_{5}\right\},\left\{v_{2}, v_{6}\right\},\left\{v_{2}, v_{7}\right\},\left\{v_{2}, v_{8}\right\},\left\{v_{2}\right.$, $\left.v_{9}\right\},\left\{v_{2}, v_{10}\right\}$ and $\left\{v_{2}, v_{12}\right\}$ are two-pairs in $G_{2}$. So there is no violation of step- 2 of algorithm-1.

(iii). The next lowest degree in the given degree sequence is 8 . Let this vertex be $v_{3}$. Since vertex $v_{3}$ has already got 2 adjacencies as can be seen in figure-1(c). Therefore we give it 6 more adjacencies by adding the edges $e_{20}, e_{21}, e_{22}, e_{23}, e_{24}$ and $e_{25}$ from vertex $v_{3}$ to $v_{4}$, $v_{5}, v_{6}, v_{7}, v_{8}$ and $v_{9}$ respectively. Doing in this way we obtain graph $G_{4}$ as shown in figure-1(d). From figure1 (c) it is clear that all the non-adjacent pairs i.e. $\left\{v_{3}\right.$, $\left.v_{4}\right\},\left\{v_{3}, v_{5}\right\},\left\{v_{3}, v_{6}\right\},\left\{v_{3}, v_{7}\right\},\left\{v_{3}, v_{8}\right\}$ and $\left\{v_{3}, v_{9}\right\}$ are two-pairs in the graph $G_{3}$. So there is again no violation of step-2 of algorithm-1.

(iv). In the given degree sequence there is another vertex of degree 8 (let it be $v_{4}$ ). Since vertex $v_{4}$ has already 3 adjacencies as can be seen in figure-1(d). So we add edges $e_{26}, e_{27}, e_{28}, e_{29}$ and $e_{30}$ from vertex $v_{4}$ to $v_{5}, v_{6}$, $v_{7}, v_{8}$ and $v_{10}$ respectively. In this way we obtain graph $G_{5}$ as shown in figure-1(e). From figure-1(d) again it is clear that all non-adjacent pairs i.e. $\left\{v_{4}, v_{5}\right\},\left\{v_{4}\right.$, $\left.v_{6}\right\},\left\{v_{4}, v_{7}\right\},\left\{v_{4}, v_{8}\right\}$ and $\left\{v_{4}, v_{10}\right\}$ are two-pairs in the graph $G_{4}$.

(v). Now the next lowest degree in the given degree sequence is 6 . Let this vertex be $v_{5}$. From figure-1(e) it is clear that vertex $v_{5}$ has already adjacency 4 , so we have to give only 2 more adjacencies in the form of edges $e_{31}$ and $e_{32}$ from vertex $v_{5}$ to $v_{6}$ and $v_{7}$ 
respectively. Thus we obtain graph $G_{6}$ in this way as shown in figure-1(f). From figure-1(e) it is clear that non-adjacent pair $\left\{v_{5}, v_{6}\right\}$ and $\left\{v_{6}, v_{7}\right\}$ are two-pairs in the graph $G_{5}$.

(vi). In the given degree sequence another vertex is of degree 6 (let it be $v_{6}$ ). Since vertex $v_{6}$ already got 5 adjacency, which can be seen in graph $G_{6}$. So we have to give only 1 adjacency in the form of adding edge $e_{33}$ from vertex $v_{6}$ to $v_{8}$. In this way we obtain graph $G_{7}$ as shown in figure-1(g). From graph $G_{6}$ it is clear that the non-adjacent pair $\left\{v_{6}, v_{8}\right\}$ is a two-pair. So adding edge between them is allowed.

As the graph $G_{7}$ has degree sequence $(10,10,8,8,6,6,5,5$, $3,3,1,1)$ which is the same degree sequence given in the input of algorithm-1. Thus algorithm stops here. Since there is no violation of step- 2 at any stage of construction, therefore the constructed graph $G_{7}$ is sc chordal on 12 vertices with degree sequence $(10,10,8,8,6,6,5,5,3,3,1,1)$.

$\mathbf{G}_{1}$

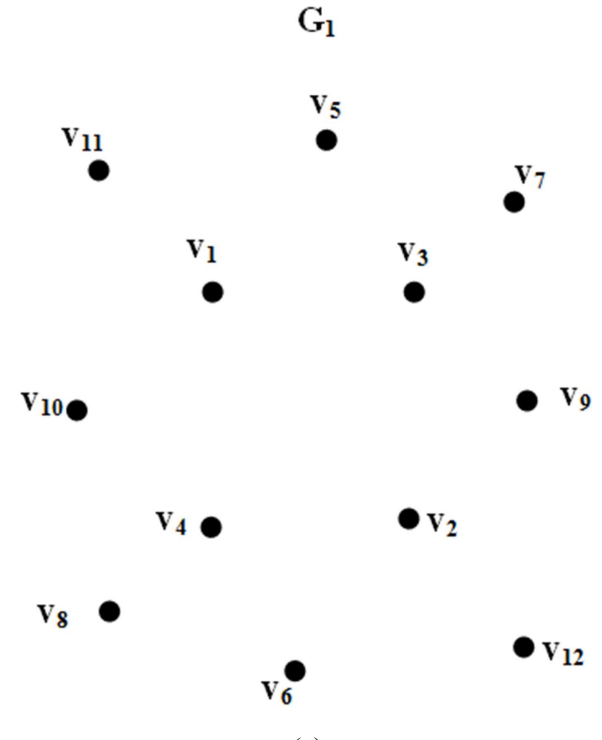

(a)

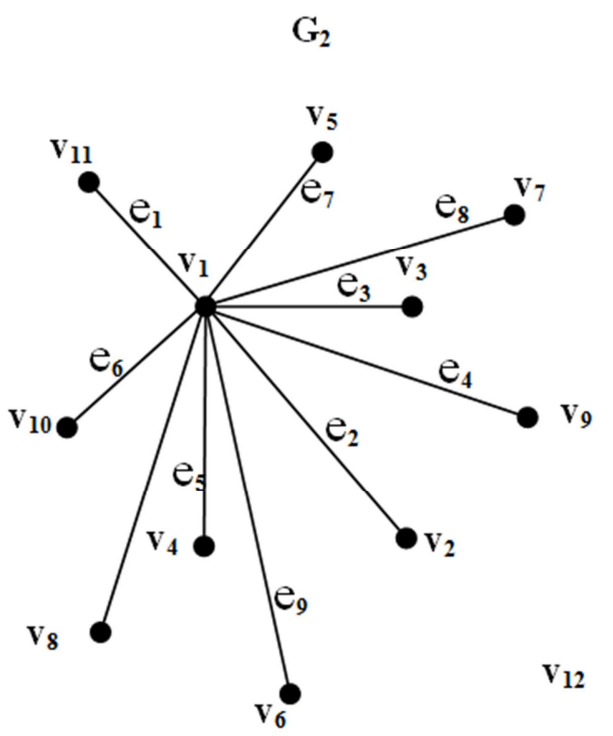

(b)

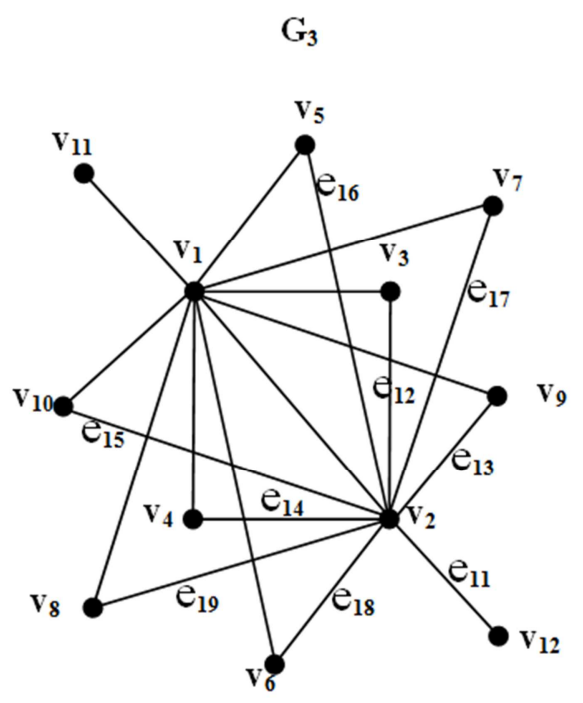

(c)

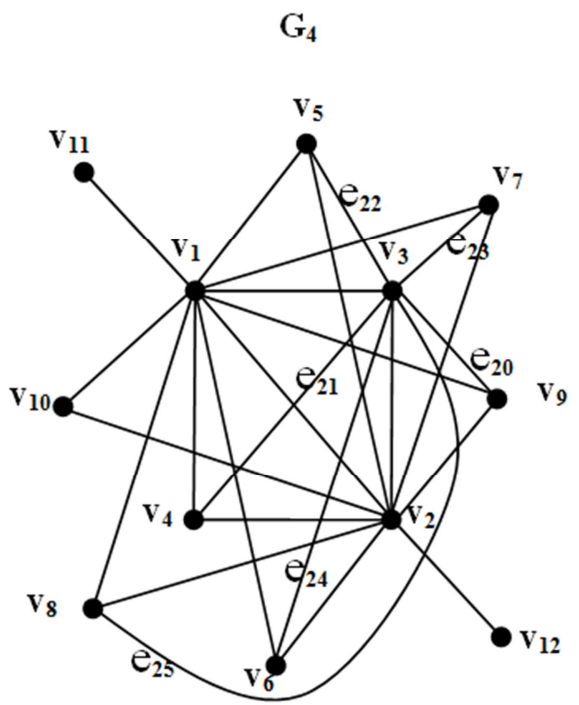

(d)

$\mathrm{G}_{5}$

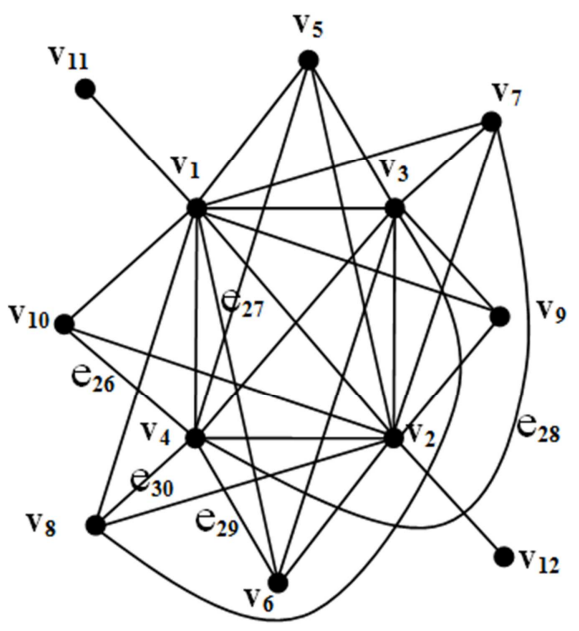

(e) 


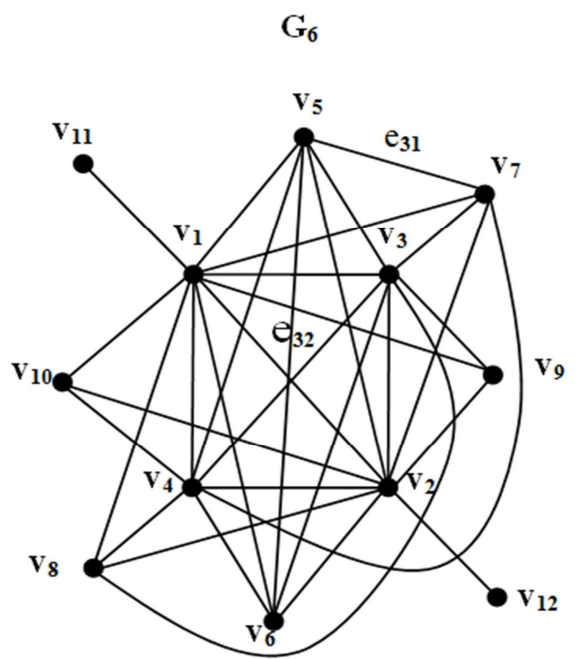

(f)

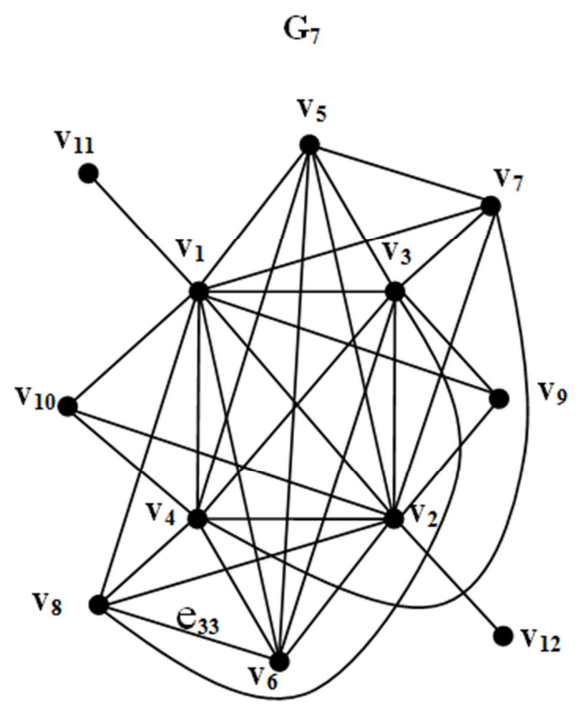

(g)

Figure 1. Construction of sc chordal graph with the given degree sequence $(10,10,8,8,6,6,5,5,3,3,1,1)$

\section{Construction of Sc Weakly Chordal Graphs}

In [12] Hayward had established a structural relationship between chordal graphs and weakly chordal graphs by defining a construction scheme on weakly chordal graphs. He noted that the class of chordal graphs can be generated by repeatedly adding a vertex which is not the middle vertex of $P_{3}$ and showed that weakly chordal graphs can likewise be generated by starting with a set of vertices and no edges and repeatedly adding an edge which is not the middle edge of $P_{4}$.

The following Theorem states Hayward [12] result in more appropriate way.

Theorem 4. A graph is weakly chordal graph iff it can be generated in the following manner

(i). Start with a graph $G_{0}$ with no edges. (ii). Repeatedly add an edge $e_{\mathrm{j}}$ to $G_{\mathrm{j}-1}$ to create the $G_{\mathrm{j}}$ such that $e_{\mathrm{j}}$ is not the middle edge of any $P_{4}$ of $G_{\mathrm{j}}$.

We give an algorithm using above result for the construction of a sc weakly chordal graph with a given degree sequence $\left(d_{1} \geq d_{2} \geq \ldots \geq d_{n}\right)$ of sc graph with $n$ vertices. The algorithm-2 work as follows: it starts with a graph $G_{\mathrm{i}}$ with no edges on $n$ vertices. Then step- 2 repeatedly adds an edge $e_{\mathrm{i}}$ between any two vertices to obtain the graph $G_{i+1}$ such that the added edge is not the middle edge of any $P_{4}$. Repeating this process if the algorithm obtains the degree sequence as given in input of the algorithm, then it stops and produces a sc weakly chordal graph.

The following algorithm constructs sc weakly chordal graph with the given degree sequence on $n$ vertices.

Algorithm 2. An Algorithm for the construction of sc weakly chordal graph.

Input: A degree sequence $\left(d_{1} \geq d_{2} \geq \ldots \geq d_{n}\right)$ which has at least one sc
graph.
Output: A sc weakly chordal graph with degree sequence
$\left(d_{1} \geq d_{2} \geq \ldots \geq d_{n}\right)$.

Step-1: Start with a graph $G_{1}$ on $n$ vertices and no edges.

Step-2: Add an edge $e_{\mathrm{i}}$ between any two vertices such that added edge $e_{\mathrm{i}}$ is not middle edge of any $P_{4}$ of $G_{\mathrm{i}}$.

Step-3: Repeat the process of step-2, until to get a graph with degree sequence $\left(d_{1} \geq d_{2} \geq \ldots \geq d_{n}\right)$.

End.

We illustrate algorithm-2 by constructing a sc weakly chordal graph on 9 vertices.

Suppose we have a degree sequence $(6,6,4,4,4,4,4,2$, 2 ) and we want to obtain a sc weakly chordal graph of this degree sequence.

Algorithm-2. starts with 9 vertices as $\left\{v_{1}, v_{2}, v_{3}, v_{4}, v_{5}, v_{6}\right.$, $\left.v_{7}, v_{8}, v_{9}\right\}$ and no edges as shown by graph $G_{1}$ in figure-2(a). We can add edges on this graph $G_{1}$ one by one such that added edge is not the middle edge of any $P_{4}$ as the rule of step-2 of algorithm-2. The overall procedure of the construction of sc weakly chordal graph with the given degree sequence $(6,6,4,4,4,4,4,2,2)$ is obtained as given below and illustrated in figure-2.

(i). The graph $G_{2}$ as shown in figure-2(b) can be easily obtained by adding edges $e_{1}, e_{2}, e_{3}, e_{4}, e_{5}$ and $e_{6}$ between the vertices $\left\{v_{1}, v_{2}\right\},\left\{v_{1}, v_{3}\right\},\left\{v_{1}, v_{4}\right\},\left\{v_{1}\right.$, $\left.v_{5}\right\},\left\{v_{1}, v_{6}\right\}$ and $\left\{v_{1}, v_{8}\right\}$ respectively. From the figure-2(b) it is clear that none of the added edges are the middle edges of any $P_{4}$.

(ii). In the graph $G_{3}$ as shown in figure-2(c), added edges are $e_{7}, e_{8}, e_{9}, e_{10}$ and $e_{11}$. Edge $e_{7}, e_{8}$ and $e_{9}$ are not the middle edges of $P_{4}$ since they are part of the triangle among the vertices $\left\{v_{1}, v_{2}, v_{3}\right\},\left\{v_{1}, v_{2}, v_{4}\right\}$ and $\left\{v_{1}, v_{2}\right.$, $\left.v_{6}\right\}$ respectively. Edges $e_{10}$ and $e_{11}$ are obviously not the middle edges of any $P_{4}$ since they have vertex $v_{7}$ and $v_{9}$ of degree 1 respectively.

(iii). In the graph $G_{4}$ as shown in figure-2(d), the added edges are $e_{12}$ and $e_{13}$. Edges $e_{12}$ and $e_{13}$ are not the middle edges of $P_{4}$ since they are part of triangle among the vertices $\left\{v_{1}, v_{3}, v_{5}\right\}$ and $\left\{v_{2}, v_{3}, v_{9}\right\}$ 
respectively.

(iv). The next graph $G_{5}$ as shown in figure-2(e) has added edges as $e_{14}$ and $e_{15}$. Both edges are the part of triangle among the vertices $\left\{v_{1}, v_{4}, v_{5}\right\}$ and $\left\{v_{2}, v_{4}, v_{7}\right\}$ respectively.

(v). In the graph $G_{6}$ as shown in figure-2(f) the only added edge is $e_{16}$. Edge $e_{16}$ is not the middle edge of any $P_{4}$ since it is part of the chordless cycle of length 4 between the vertices $v_{2}, v_{3}, v_{5}$, and $v_{7}$.

(vi). The next graph $G_{7}$ as shown in figure-2(g) has added edges as $e_{17}$ and $e_{18}$. Both edges are the part of triangle among the vertices $\left\{v_{2}, v_{6}, v_{7}\right\}$ and $\left\{v_{1}, v_{6}, v_{8}\right\}$ respectively.

The algorithm-2 stops here, since graph $G_{7}$ has degree sequence $(6,6,4,4,4,4,4,2,2)$ which is the same degree sequence as given in input of the algorithm. Since there is no violation at any stage of the algorithm, so the constructed graph $G_{7}$ is sc weakly chordal graph with the degree sequence $(6,6,4,4,4,4,4,2,2)$ on 9 vertices.

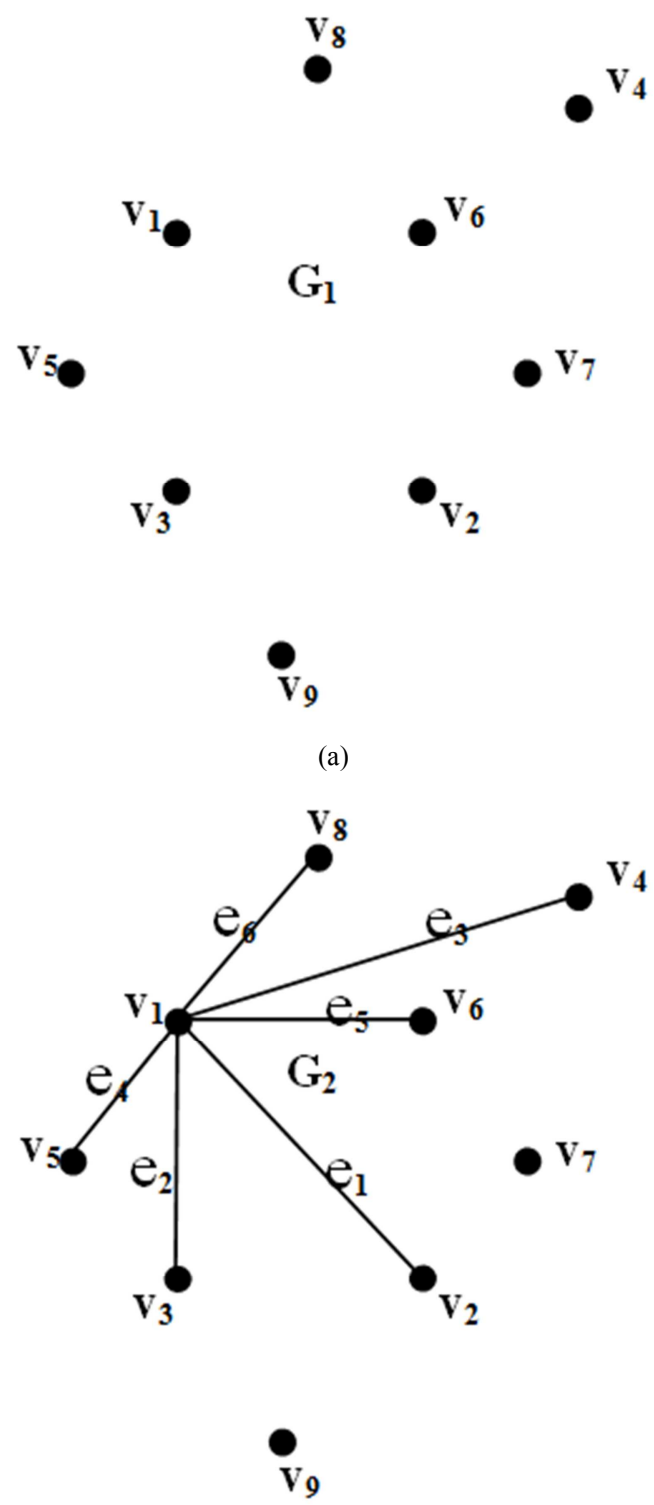

(b)

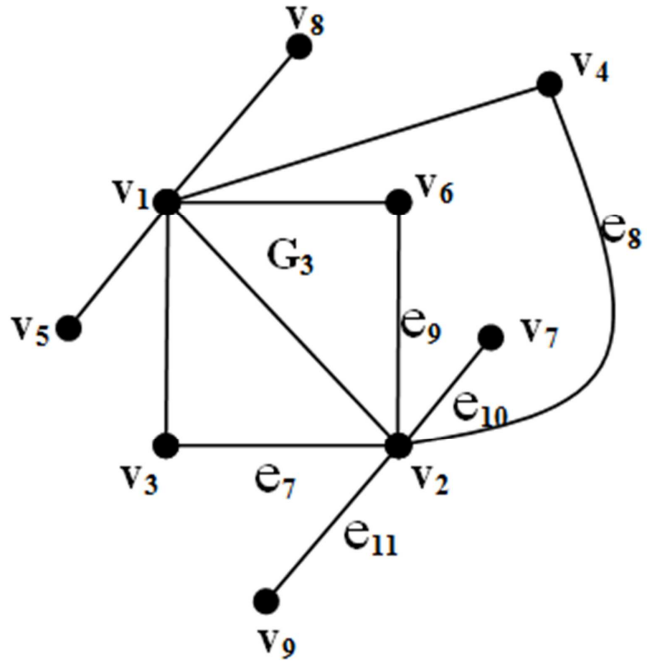

(c)

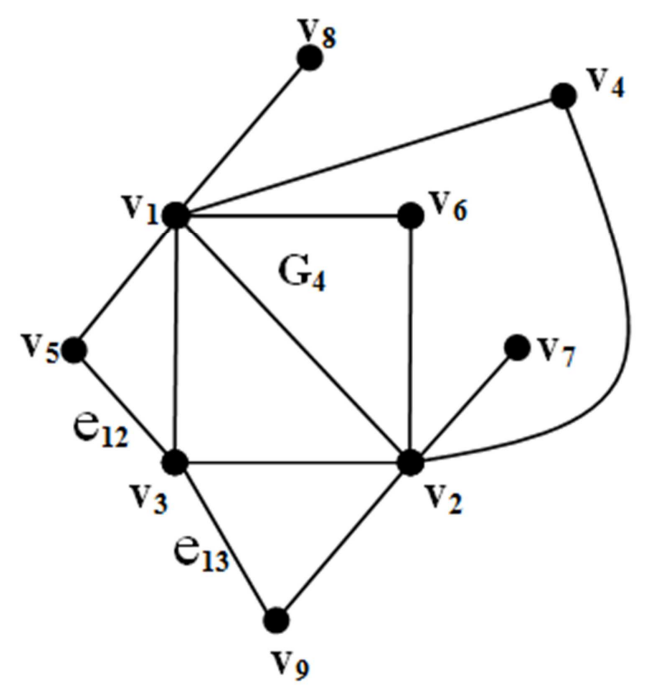

(d)

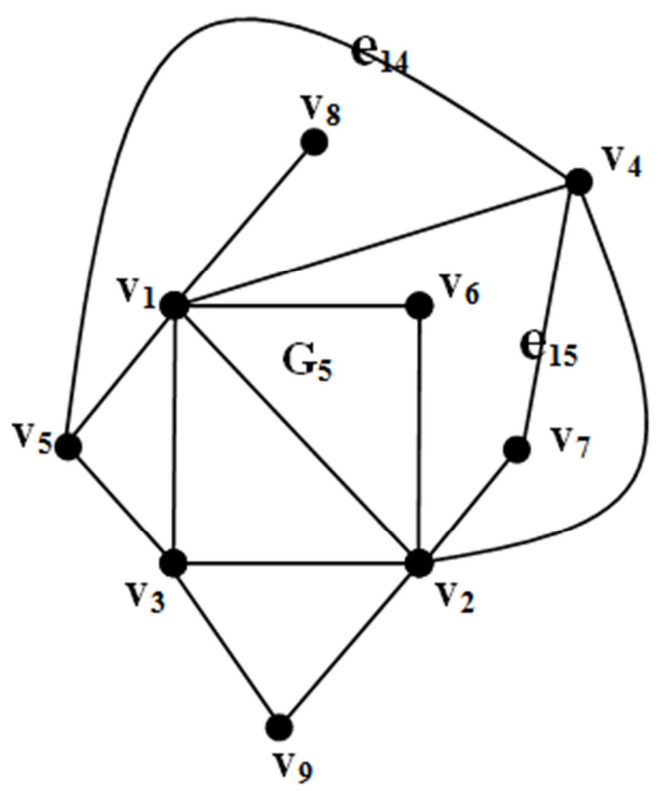

(e) 


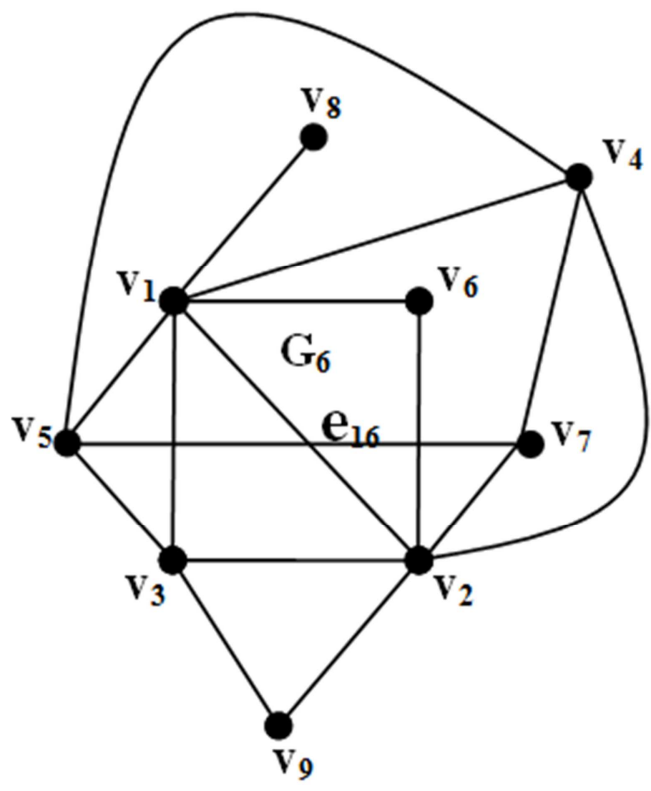

(f)

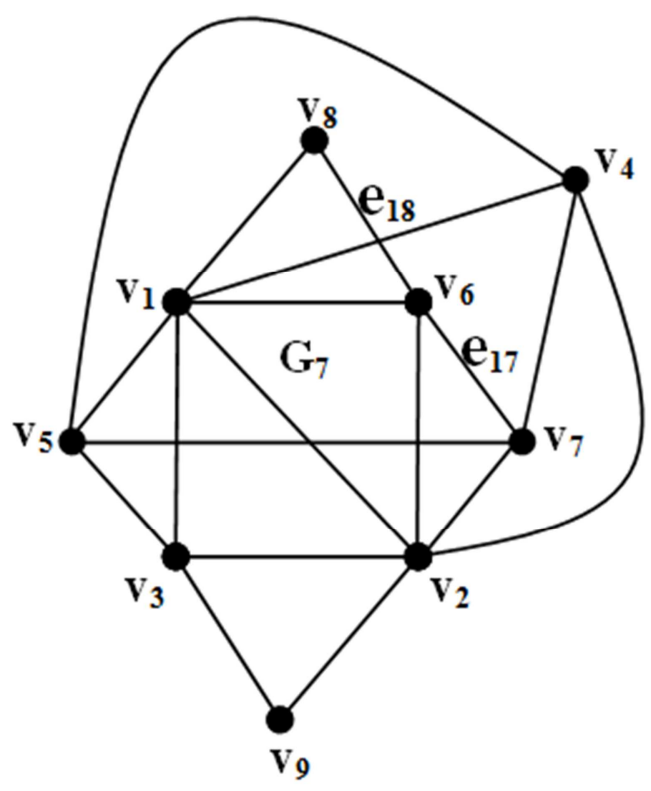

(g)

Figure 2. Construction of sc weakly chordal graph with the given degree sequence $(6,6,4,4,4,4,4,2,2)$.

\section{Conclusion}

In [10] the given algorithms construct the same graph many times. So in order to, compile a catalogue of sc chordal graphs with $4 p+1$ vertices, every pair of the constructed graphs should be tested whether the graphs are isomorphic or not after constructing all sc chordal graphs with $4 \mathrm{p}+1$ vertices from the given algorithms.

The algorithm- 1 given in section 2 takes input a degree sequence of sc graph and throughout the process the chordality of graphs is not violated. Also from theorem- 1 no other graph is possible with the same degree sequence. So it is eay to construct all the sc chordal graph by the algorithm-1 as compare to algorithm given in [10].

\section{References}

[1] A. Berry, A. Sigayret and C. Sinoquet, Maximal subtriangulation as pre-processing phylogentic data, Soft computing 10 (2006) 461-468.

[2] C. T. Hoang, Brain Moore, D. Roecoskie, J Sawada, M. Vatshelle, Construction of $k$ - critical $P_{5}$-free graphs, Discrete Applied Mathematics, 182 (2015), 91-98.

[3] D. R. Fulkerson and O. A. Gross, Incidence matrices and interval graphs, Pacafic J. Math., 15 (1965) 835-855.

[4] G. Ringel, Selbstkomplementare Graphen, Arch Math. (Basel), 14 (1963) 354-358.

[5] H. Sachs, Uber selbstkomplementare Graphen, Publ. Math. Debreen, 9 (1962) 270-288.

[6] J. Yellen and J Gross, Graph Theory and its Applications, CRC Press (USA), 1999.

[7] J. Xu and C. K. Wong, Self-complementary graphs and Ramsey numbers Part-1: the decomposition and the construction of self-complementary graphs, Discrete mathematics, 223 (2000) 309-326.

[8] Lihuan Mao, Fenjin Liu, Wei Wang, A new method for the constructing graphs determined by their generalized spectrum, Linear Algebra and its Applications, 477 (2015) 112-127.

[9] M. R. Sridharan and K. Balaji, Characterisation of selfcomplementary chordal graphs, Discrete Mathematics, 188 (1998) 279-283.

[10] M. R. Sridharan and K. Balaji, On construction of selfcomplementary chordal graphs, Journal of Combinatorics Information and system sciences, 24 (1999) 39-45.

[11] R. A. Gibbs, Self-Complementary Graphs, J. Comb. Theory Series B, 16 (1974) 106-123.

[12] R. B. Hayward, Generating weakly triangulated graphs, J. Graph Theory, 21 (1996) 67-70.

[13] Xueyi Huang and Qiongxiang Huang Construction of graphs with exactly $k$ main eigenvalues, Linear Algebra and its Applications, 486 (2015), 204-218 